\section{NICE recommends faster, easier access to care for MS patients}

Anjali Jain BMJ

Every patient who is suspected of having multiple sclerosis should be seen by a neurologist within six weeks of referral, and investigations should be completed within another six weeks. These are recommendations in the new guidelines of the National Institute for Clinical Excellence (NICE) that were issued this week.

The aim of the guidelines is to provide easier and faster access to specialised services for the 50000 to 60000 people with the condition. The guidelines were welcomed by patient groups.

The institute laid down six key priorities, including reducing delays in diagnosis and producing a responsive and seamless service from the viewpoint of the patient.

Specialist services should be available to everyone when they needed them, which was usually when they developed any new symptom or sign. Patients should be able to refer themselves to a specialist in such circumstances.

Every health commissioning organisation, primarily primary care trusts, should ensure that all organisations in a local heath area agree and publish protocols and procedures for sharing and transferring clinical and resource responsibility for people with multiple sclerosis.

In addition, health professionals should consider in a systematic way whether the person with multiple sclerosis had any sensitive and hidden problems that could worsen their condition, including depression, fatigue, and sexual dysfunction.

The institute recommended that the number of UK neurologists be tripled, from the current 360 , and the number of nurse specialists doubled, from the current 150. It also made evidence based suggestions for the treatment of acute episodes with steroids and described the possible benefit of some complementary therapies such as fish oils and reflexology.

Andrew Dillon, the institute's chief executive, who described the guidelines as "aspirational but also achievable," admitted that they had not been costed.

The recommendations were welcomed by several patient groups. Mike O'Donovan, chief executive of the Multiple Sclerosis Society, said: "This is a very big day for people with multiple sclerosis."

A spokesman for the charity MS Trust said: "The introduction of a clinical guideline for the management of MS, which provides a benchmark for good practice in the NHS, will undoubtedly bring further benefits to people with MS."

Copies of the guidelines (including a version for patients and families) are available from the NHS Response Line (tel 08701555 455) or from the NICE website (www.nice.org.uk).

\section{Scientists find gene linking inherited breast and ovarian cancer to non-inherited cases}

\section{Susan Mayor London}

A new gene for breast and ovarian cancer has been identified, which explains the link between hereditary and "sporadic" (noninherited) forms of these cancers.

It has been known for some time that mutations in the BRCA1 and BRCA2 genes are associated with inherited breast and ovarian cancer. However, only $5 \%$ of breast cancers are inherited and no role had been found for these genes in noninherited cancers.

Researchers have been hunting for the genes associated with sporadic breast cancers $-95 \%$ of cases-ever since the BRCA genes were first identified, eight years ago.

Research published this week has identified a new gene, EMSY, which binds to normally functioning BRCA2, potentially triggering the development of cancer (Cell 2003;115:523-5).

EMSY was isolated by researchers from the Wellcome Trust/Cancer Research UK Institute, at the University of Cambridge, when they were looking for sequences of DNA that interacted with BRCA2. The new gene proved to have a role in switching genes on and off and in repairing damaged DNA-relocating to sites of DNA damage.

It was found to be particularly effective at switching off BRCA2, raising suspicions that it might be an important cancer gene. This suspicion was strengthened when colleagues at the Cancer Research UK's department of oncology at Cambridge University found extra copies of EMSY in the first few tumour samples that they tested.

Working with a team at the British Columbia Cancer Agency in Canada, the Cambridge group then analysed EMSY in more than 500 breast and 300 ovarian tumours. They found that the gene was amplified in 13\% of breast cancers and $17 \%$ of ovarian cancers, but never in normal tissue and very rarely in other types of tumour.

One of the research group, Dr Luke Hughes-Davies, a Cancer Research UK clinical scientist at Addenbrooke's Hospital, Cambridge, said: "The remarkable clinical overlap between sporadic EMSY amplification and familial BRAC2 deletion- which only occurs in breast and ovarian tumours-implicates a BRCA2 pathway in sporadic breast and ovarian cancers.'

The discovery of EMSY has several important clinical implications. EMSY amplification was found to be associated with a poor prognosis, particularly in node-negative breast cancer. Women whose tumours showed increased expression of EMSY had an average survival of 6.4 years, compared with 14 years for women whose breast cancers showed normal levels of EMSY. This suggests that it might be of prognostic value. The gene also offers a new target for the devel-

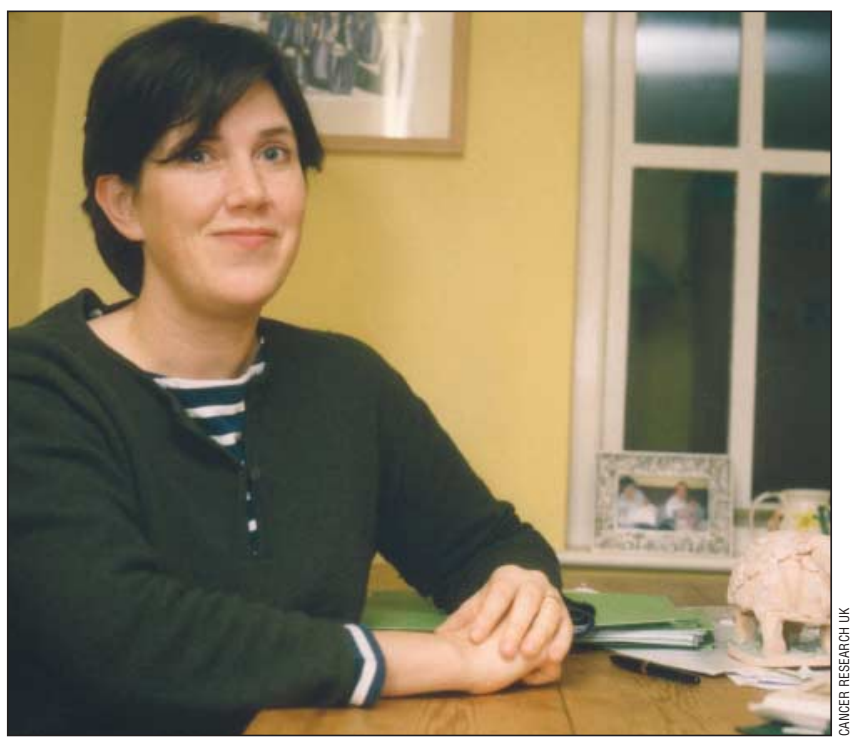

The new gene EMSY is named after breast cancer nurse Emma Hughes-Davies (above), who was called Emsy when she was a child 